1 Universidade Federal de Pernambuco (UFPE), Programa de PósGraduação em Saúde Coletiva - Recife (PE) Brasil.

elisamouramelo@gmail.com

2 Universidade Federal de Pernambuco (UFPE)

Programa de PósGraduação em Saúde Coletiva e Departamento de Medicina Social - Recife (PE), Brasil.

marquesap@hotmail.com

3 Universidade Federal de Pernambuco (UFPE), Programa de Pós-

Graduação em Saúde Coletiva e Departamento de Medicina Social - Recife $(\mathrm{PE})$, Brasil.

marciacarrera@hotmail.com

4 Universidade Federal de Pernambuco (UFPE) Núcleo de Ciências da Vida, Curso de Medicina Caruaru (PE), Brasil.

hugomouramelo@gmail.com

\section{Síndrome da fragilidade e fatores associados em idosos residentes em instituições de longa permanência}

\author{
Frailty syndrome and associated factors in elderly residents in long- \\ term institutions
}

Elisa Moura de Albuquerque Melo', Ana Paula de Oliveira Marques², Márcia Carrera Campos Leal'3, Hugo Moura de Albuquerque Melo4

DOI: 10.1590/0103-1104201811710

RESUMO Estudo descritivo em instituições de longa permanência para investigar a prevalência de fragilidade e de fatores associados em idosos institucionalizados e sua associação com variáveis sociodemográficas e condições de saúde. Foram investigados 214 idosos, a maioria do sexo feminino (69,6\%), estado civil solteiro (53,7\%), escolaridade de um ano $(54,4 \%)$ e renda de até um salário mínimo (73,4\%). Quanto à cognição, 79,4\% foram reprovados por erros significativos. A síndrome de fragilidade foi identificada em 70,1\% dos idosos. Todos os fatores incluídos na escala de fragilidade alcançaram significância estatística. Na análise multivariada, os fatores que mais contribuem para a fragilidade são: cognição, independência funcional, autoavaliação de saúde, frequência de suporte social, percepção de perda de peso e depressão.

PALAVRAS-CHAVE Idoso. Instituição de longa permanência para idosos. Idoso fragilizado. Saúde do idoso institucionalizado.

\begin{abstract}
Descriptive study in long-term institutions to investigate the prevalence of frailty and associated factors in institutionalized elderly and its association with sociodemographic variables and health conditions. A total of 214 elderly people, most of them females (69.6\%), single marital status (53.7\%), one year of schooling (54.4\%) and income of up to a minimum wage (73.4\%) were investigated. As to cognition, $79.4 \%$ were disapproved for significant errors. Frailty syndrome was identified in $70.1 \%$ of the elderly. All the factors included in the fragility scale reached statistical significance. In the multivariate analysis, the factors that contribute most to the fragility are: cognition, functional independence, health self-assessment, frequency of social support, perception of weight loss and depression.
\end{abstract}

KEYWORDS Aged. Homes for the aged. Frail elderly. Health of institutionalized elderly. 


\section{Introdução}

O acelerado processo de envelhecimento populacional e o aumento da expectativa de vida devem despertar a atenção da sociedade para as condições de saúde, morbidade e limitações funcionais dos idosos. As mudanças observadas na estrutura demográfica resultam em alterações no perfil epidemiológico, com redução das doenças infectocontagiosas e aumento das doenças crônico-degenerativas, que, em sua maioria, interferem na capacidade funcional e na qualidade de vida da população que envelhece'.

Nesse contexto, a síndrome da fragilidade do idoso tem sido conceituada como uma condição clinicamente diagnosticável, resultante do declínio das reservas fisiológicas e funcionais em diversos sistemas, proporcionando menor tolerância fisiológica e psicológica aos estressores e exposição a risco elevado de eventos adversos à saúde física e mental ${ }^{2}$.

Após diversas tentativas internacionais de estabelecer parâmetros clínicos para o diagnóstico da síndrome da fragilidade, encontra-se na literatura o seguinte consenso: a) é uma síndrome clínica; b) indica aumento da vulnerabilidade aos estressores, acarretando prejuízos funcionais e aumento dos eventos adversos à saúde; c) pode ser reversível ou atenuada por intervenções; d) seu conhecimento é útil para planejamento e realização de cuidados primários ${ }^{3}$.

O consenso internacional vigente admite que os aspectos biológico e social devem integrar o cuidado ao idoso, uma vez que o crescimento da fragilidade em uma população ocorre de modo proporcional ao maior número de idosos ${ }^{2}$. Esse fato caracteriza o tema como um importante problema de saúde pública, pois o aumento da expectativa de vida fará com que cada vez mais idosos precisem de cuidados ${ }^{4}$.

O aumento na demanda por institucionalização de idosos em Instituições de Longa Permanência para Idosos (Ilpi) constitui-se um desafio social que merece atenção. As alterações próprias do envelhecimento e as doenças preexistentes podem ser agravadas pelas dificuldades de adaptação desses indivíduos às novas condições de vida que a institucionalização lhes impõe, o que pode contribuir para maior vulnerabilidade à fragilidade e ao declínio funcional ${ }^{5}$.

Por considerar a importância do tema, o presente artigo foi baseado em estudo que teve por hipótese haver maior fragilidade entre os idosos residentes em instituições de longa permanência e teve por objetivo investigar a prevalência da síndrome da fragilidade em idosos institucionalizados e sua associação com variáveis sociodemográficas e condições de saúde.

\section{Métodos}

Trata-se de um estudo descritivo, quantitativo de corte transversal. A pesquisa foi desenvolvida em nove Ilpi, sendo duas de administração pública e sete filantrópicas, regulamentadas pela prefeitura da cidade do Recife, estado de Pernambuco.

A população correspondeu a 432 idosos, de ambos os sexos, com idade equivalente ou superior a 60 anos, residentes nas Ilpi selecionadas. Os critérios de inclusão adotados corresponderam a: não apresentar diagnóstico sugestivo de demência, com base no resultado do Miniexame do Estado Mental ou comprometimento de comunicação que pudesse interferir na obtenção dos dados, resultando em uma população elegível correspondente a 214 idosos após as exclusões. Por se tratar de um estudo em que foram avaliados vários aspectos relacionados com as condições de saúde dos idosos institucionalizados, optou-se por incluir aqueles que atenderam aos critérios de inclusão estabelecidos.

A variável dependente consistiu da graduação da fragilidade do idoso, avaliada por meio da Escala de Fragilidade de Edmonton ${ }^{6}$ que utiliza como critérios a avaliação 
geriátrica ampla considerando cognição, diagnosticada pelo teste do desenho do relógio; equilíbrio e imobilidade avaliados pelo Teste Get Up and Go; além de humor, independência funcional, uso de medicação, suporte social, nutrição, atitude saudáveis, continência urinária e presença de morbidades diagnosticadas.

Como variáveis independentes, foram considerados: dados sociodemográficos e condições de saúde autorreferidas obtidas em entrevista semiestruturada, além dos diagnósticos e informações sobre uso das medicações que foram coletados por fonte secundária, no prontuário do idoso na Ilpi em que residia. As informações foram cuidadosamente coletadas, e, em casos dúbios, houve consulta a equipe de saúde para esclarecimentos, minimizando, assim, possível viés de memória.

Os dados foram coletados no período de janeiro a maio de 2013 , com registro das informações em protocolo semiestruturado. As entrevistas foram realizadas por uma equipe de cinco alunas do mestrado em saúde coletiva, previamente treinadas com discussões sobre os instrumentos e aplicação interpares. Para análise de coerência do roteiro de entrevista, foi realizado um estudo piloto, por seleção aleatória, em $10 \%$ dos idosos residentes nas Ilpi investigadas. Durante a coleta das informações relacionadas com a fragilidade, constatou-se que 61 idosos não sabiam ler as horas, motivo que os impossibilitou de realizar o teste de desenho do relógio.

A análise descritiva foi realizada por meio de frequências absolutas e relativas. Para determinação da razão de prevalência, foi realizada análise de regressão logística bivariada, as variáveis que alcançaram significância em nível de 0,20 foram incluídas no modelo de regressão logística multivariado, determinando-se as razões de prevalência brutas e ajustadas, em nível de significância de 5\%.

A pesquisa desenvolvida esteve vinculada ao projeto intitulado 'Perfil social e epidemiológico de pessoas idosas no contexto asilar', registrado no Comitê de Ética em Pesquisa da Universidade Federal de Pernambuco sob CAAE n ${ }^{\circ}$ 02013112600005208. As instituições tiveram sua participação validada por autorização de seus responsáveis técnicos, e os idosos foram entrevistados apenas após estar de acordo e assinar termo de consentimento livre e esclarecido. Todo o processo de investigação atendeu aos requisitos estabelecidos na Resolução $n^{0} 466$, de 12 de outubro de 2012, do Conselho Nacional de Saúde.

\section{Resultados}

Do total de idosos investigados $(\mathrm{n}=214)$, a média de idade correspondeu a 76,4, variando de 60 a 104 anos (tabela 1).

Tabela 1. Distribuição de frequência das variáveis independentes de idosos residentes em Ilpi. Recife, PE, 2013

\begin{tabular}{lr}
\hline Variável & $\mathbf{n ( \% )}$ \\
\hline Sexo & $149(69,6)$ \\
\hline Feminino & $65(30,4)$ \\
Masculino & $60(28,0)$ \\
\hline Faixa etária (anos) & $77(36,0)$ \\
\hline $60-69$ & $56(26,2)$ \\
$70-79$ & $21(9,8)$ \\
\hline $80-89$ & ou mais
\end{tabular}


Tabela 1. (cont.)

\begin{tabular}{|c|c|}
\hline Estado conjugal ${ }^{\star}$ & \\
\hline Solteiro & $115(54,0)$ \\
\hline Viúvo & $56(26,3)$ \\
\hline Separado/divorciado & $24(11,2)$ \\
\hline Com companheiro ${ }^{\star *}$ & $18(8,5)$ \\
\hline \multicolumn{2}{|l|}{ Educação formal } \\
\hline Sim & $160(74,8)$ \\
\hline Não & $54(25,2)$ \\
\hline \multicolumn{2}{|l|}{ Tempo de estudo (anos) } \\
\hline $1-4$ & $87(54,4)$ \\
\hline $5-8$ & $28(17,5)$ \\
\hline $9-11$ & $17(10,6)$ \\
\hline 12 ou mais & $8(5,0)$ \\
\hline Não sabe informar & $20(12,5)$ \\
\hline \multicolumn{2}{|c|}{ Situação financeira/previdenciária } \\
\hline Sem renda & $29(13,6)$ \\
\hline Aposentado & $163(76,2)$ \\
\hline Pensionista & $17(7,9)$ \\
\hline Aposentado e pensionista & $5(2,3)$ \\
\hline \multicolumn{2}{|c|}{ Renda pessoal (em salário mínimo) $†$} \\
\hline Sem renda & $29(14,1)$ \\
\hline Até 1 & $157(76,2)$ \\
\hline$>1-2$ & $14(6,8)$ \\
\hline$>2-4$ & $5(2,4)$ \\
\hline$>4$ & $1(0,5)$ \\
\hline \multicolumn{2}{|l|}{ Natureza da llpi } \\
\hline Pública & $30(14,0)$ \\
\hline Filantrópica & $184(86,0)$ \\
\hline \multicolumn{2}{|c|}{ Tempo de institucionalização (anos) } \\
\hline$<1$ & $63(29,4)$ \\
\hline $1-3$ & $66(30,8)$ \\
\hline $3-5$ & $27(12,6)$ \\
\hline$>5$ & $58(27,1)$ \\
\hline
\end{tabular}

*Um (0,5\%) idoso omitiu estado conjugal; ${ }^{* *}$ com companheiro remete aos idosos institucionalizados, apesar de ainda terem companheiros vivos; †'oito (3,7\%) idosos omitiram a informação de renda pessoal.

A distribuição e a frequência dos fatores considerados na avaliação da fragilidade dos idosos são descritas na tabela 2. Para análise da medida de associação, a variável dependente foi categorizada em 64 idosos não frágeis (29,9\%), incluídos nessa categoria os não frágeis e vulneráveis, e 150 (70,1\%) considerados frágeis. 
Tabela 2. Distribuição de frequência das condições de saúde de idosos residentes em Ilpi. Recife, PE, 2013

\begin{tabular}{lr}
\hline Variável & $\mathbf{n}(\%)$ \\
\hline Avaliação da cognição & $28(13,1)$ \\
\hline Aprovado & $16(7,5)$ \\
Reprovado com erros mínimos & $170(79,4)$ \\
Reprovado com erros significativos†t & $169(79,0)$ \\
Número de internamentos hospitalares & $39(18,2)$ \\
Nenhum & $6(2,8)$ \\
\hline $1-2$ & $88(41,3)$ \\
$>2$ & $79(37,1)$ \\
\hline Autoavaliação da saúde & \\
\hline Excelente a boa & $46(21,6)$ \\
Razoável &
\end{tabular}

Número de $A V D^{\star \star}$ comprometidas

No máximo uma

Duas a quatro

Cinco a oito

$106(49,5)$

Disponibilidade de suporte social

Sempre

$147(68,7)$

Algumas vezes

$45(21,0)$

Nunca

$22(10,3)$

Uso de cinco ou mais medicamentos

Não

Sim

$84(39,4)$

Esquece de tomar o medicamento

Não

Sim

$41(19,2)$

Autorreferência de perda de peso recente

Não

Sim

Sentimento de tristeza ou depressão

Não

Sim

$120(56,1)$

Incontinência urinária

Não

Sim

$94(43,9)$

Teste Get Up and Go

0-10 segundos

11-20 segundos

$>20$ segundos

† Teste do relógio estratificado conforme classificação padronizada pela escala de Edmonton8; *Um (0,5\%) idoso não informou a autoavaliação de saúde e outro omitiu informação; **AVD: atividades de vida diária; ††'Cinco (2,3\%) idosos não informaram perda de peso recente. 
Na tabela 3, estão expressas as associações das variáveis analisadas com a presença de síndrome de fragilidade. Quanto ao comprometimento da cognição, a reprovação por erros significativos no teste do relógio aumentou a razão de prevalência em 10 vezes (IC95\% 4,1-24,6). Constatou-se que todos os fatores incluídos na escala de fragilidade do idoso alcançaram significância estatística associada ao aumento da razão de prevalência.

Tabela 3. Fatores associados à síndrome da fragilidade de idosos residentes em Ilpi. Recife, PE, 2013

\begin{tabular}{|c|c|c|c|c|c|}
\hline \multirow{2}{*}{ Variáveis relativas à fragilidade } & Amostra & Não frágil & Frágil & \multirow{2}{*}{ RP (IC95\%) } & \multirow{2}{*}{ Valor $\mathrm{p}$} \\
\hline & $\mathrm{n}$ & n (\%) & n (\%) & & \\
\hline Cognição & & & & & $<0.001$ \\
\hline Aprovado & 28 & $20(71,4)$ & $8(28,6)$ & 1,0 & \\
\hline Reprovado com erros mínimos & 16 & $10(62,5)$ & $6(37,5)$ & $1,5(0,5-5,2)$ & \\
\hline Reprovado com erros significativos & 170 & $34(20,0)$ & $136(80,0)$ & $10,0(4,1-24,6)$ & \\
\hline Número de internamentos & & & & & 0,004 \\
\hline Nenhum & 169 & $59(34,9)$ & $110(65,1)$ & 1,0 & \\
\hline Um ou mais & 45 & $5(11,1)$ & $40(88,9)$ & $4,3(1,6-11,4)$ & \\
\hline Autoavaliação da saúde ${ }^{\star}$ & & & & & $<0.001$ \\
\hline Excelente a boa & 88 & $46(52,3)$ & $42(47,7)$ & 1,0 & \\
\hline Razoável & 79 & $16(20,3)$ & $63(79,7)$ & $4,3(2,2-8,6)$ & \\
\hline Ruim & 46 & $2(4,3)$ & $44(95,7)$ & $24,1(5,5-105,6)$ & \\
\hline Número de AVD ${ }^{\star \star}$ comprometidas & & & & & $<0.001$ \\
\hline $0-1$ & 50 & $33(66,0)$ & $17(34,0)$ & 1,0 & \\
\hline $2-4$ & 58 & $20(34,5)$ & $38(65,5)$ & $3,7(1,7-8,2)$ & \\
\hline $5-8$ & 106 & $11(10,4)$ & $95(89,6)$ & $16,8(7,1-39,4)$ & \\
\hline Disponibilidade de suporte social & & & & & 0,003 \\
\hline Sempre & 147 & $55(37,4)$ & $92(62,6)$ & 1,0 & \\
\hline Algumas vezes & 45 & $5(11,1)$ & $40(88,9)$ & $4,8(1,8-12,8)$ & \\
\hline Nunca & 22 & $4(18,2)$ & $18(81,8)$ & $2,7(0,9-8,4)$ & \\
\hline Uso de cinco ou mais medicamentos & & & & & $<0.001$ \\
\hline Não & 129 & $51(39,5)$ & $78(60,5)$ & 1,0 & \\
\hline $\operatorname{sim}$ & 84 & $13(15,5)$ & $71(84,5)$ & $3,6(1,8-7,1)$ & \\
\hline Esquece de tomar medicamento & & & & & 0,022 \\
\hline Não & 173 & $58(33,5)$ & $115(66,5)$ & 1,0 & \\
\hline Sim & 41 & $6(14,6)$ & $35(85,4)$ & $2,9(1,2-7,4)$ & \\
\hline Autorreferência de perda de peso recente & & & & & $<0.001$ \\
\hline Não & 129 & $52(40,3)$ & $77(59,7)$ & 1,0 & \\
\hline Sim & 80 & $10(12,5)$ & $70(87,5)$ & $4,7(2,2-10,0)$ & \\
\hline Sentimento de tristeza/depressão & & & & & $<0.001$ \\
\hline Não & 94 & $46(48,9)$ & $48(51,1)$ & 1,0 & \\
\hline Sim & 120 & $18(15,0)$ & $102(85,0)$ & $5,4(2,8-10,3)$ & \\
\hline Incontinência urinária & & & & & $<0.001$ \\
\hline Não & 120 & $58(48,3)$ & $62(51,7)$ & 1,0 & \\
\hline Sim & 94 & $6(6,4)$ & $88(93,6)$ & $13,7(5,3-33,8)$ & \\
\hline
\end{tabular}


Tabela 3. (cont.)

\begin{tabular}{|c|c|c|c|c|c|}
\hline Teste Get Up and Go & & & & & $<0.001$ \\
\hline $0-10$ segundos & 18 & $13(72,2)$ & $5(27,8)$ & 1,0 & \\
\hline $11-20$ segundos & 62 & $30(48,4)$ & $32(51,6)$ & $2,8(0,9-8,7)$ & \\
\hline$>20$ segundos & 134 & $21(15,7)$ & $113(84,3)$ & $14,0(4,5-43,4)$ & \\
\hline Tempo de institucionalização (anos) & & & & & 0.898 \\
\hline$<3$ & 129 & $39(30,2)$ & $90(69,8)$ & $1,0(0,5-1,8)$ & \\
\hline$\geq 3$ & 85 & $25(29,4)$ & $60(70,6)$ & 1,0 & \\
\hline Faixa etária (anos) & & & & & 0.120 \\
\hline 60 a 79 & 137 & $46(33,6)$ & $91(66,4)$ & 1,0 & \\
\hline 80 ou mais & 77 & $18(23,4)$ & $59(76,6)$ & $1,6(0,9-3,1)$ & \\
\hline Tempo de estudo (anos) & & & & & 0.047 \\
\hline Não estudou & 54 & $11(20,4)$ & $43(79,6)$ & $3,0(1,3-6,6)$ & \\
\hline Um a quatro & 61 & $14(23,0)$ & $47(77,0)$ & $2,5(1,2-5,3)$ & \\
\hline Mais de quatro & 79 & $34(43,0)$ & $45(57,0)$ & 1.0 & \\
\hline Situação financeira & & & & & 0.063 \\
\hline Aposentado/pensionista & 185 & $13(44,8)$ & $16(55,2)$ & $0,5(0.2-1.0)$ & \\
\hline Sem renda & 29 & $51(27,6)$ & $134(72,4)$ & 1.0 & \\
\hline Renda pessoal (em salário mínimo) & & & & & 0.034 \\
\hline Sem renda & 29 & $13(44,8)$ & $16(55,2)$ & $2,0(1,0-4,0)$ & \\
\hline Renda de até 1 & 157 & $42(26,8)$ & $115(73,2)$ & $1,6(0,6-4,1)$ & \\
\hline Renda maior que 1 & 20 & $8(40,0)$ & $12(60,0)$ & 1,0 & \\
\hline
\end{tabular}

Dessa forma, a presença de um ou mais internamentos aumentou a razão de prevalência em 4,3 vezes (IC 95\% 1,6-11,4). A piora na autoavaliação de saúde esteve associada ao aumento da razão de prevalência de 4,3 vezes (IC 95\% 2,2-8,6), quando a avaliação era 'razoável', para 21,1 vezes (IC 95\% 5,5-105,6), quando a saúde era percebida como 'ruim'.

Em relação ao comprometimento das Atividades de Vida Diária (AVD), houve aumento da razão de prevalência de 3,7 vezes (IC95\% 1,7-8,2), quando a execução de duas a quatro AVD estava dificultada, e para 16,8 vezes (IC95\% 7,1-39,4), se cinco a oito não podiam ser realizadas pelo idoso sem ajuda. No que diz respeito ao suporte social, sua disponibilidade restrita a algumas vezes aumentou a razão de prevalência em 4,8 vezes (IC95\% 1,8-12,8), mas a inexistência desse apoio não interferiu sobre a razão de prevalência.
Quanto à medicação, o uso de cinco ou mais fármacos assim como a referência ao esquecimento de tomá-los aumentaram a razão de prevalência em aproximadamente três vezes (tabela 3 ).

A autorreferência de perda de peso recente e o sentimento de tristeza ou depressão também promoveram aumento da razão de prevalência em cerca de cinco vezes. A referência de incontinência urinária aumentou em 13,7 vezes (IC95\% 5,3-33,8) a razão de prevalência de fragilidade, tal como se verificou quando o retardo no tempo para completar o Teste Get Up and Go foi maior que 20 segundos, pois a razão de prevalência igualou-se a 14,0 (IC 95\% 4,5-43,4).

Entre as variáveis sociodemográficas, exclusivamente, o tempo de estudo e a renda pessoal relacionaram-se significantemente com a fragilidade. A escolaridade restrita de 
um a quatro anos contribuiu significantemente para a fragilidade do idoso, com razão de prevalência igual a 3,0 (IC95\% 1,3-6,6), quando o idoso declarou não ter estudo, e 2,5 (IC95\% 1,2-5,3), quando tinha de um a quatro anos de estudo. Quanto à renda pessoal, identificou-se que sua ausência aumentou a razão de prevalência em duas vezes (IC95\% 1,0-4,0), (tabela 3).

A partir da análise bivariada, foi construída a análise multivariada, cujos resultados estão expressos na tabela 4, na qual se pode identificar que os fatores que mais contribuíram para a prevalência foram o comprometimento da cognição, cujo agravamento registra maior prevalência da fragilidade, e o prejuízo na independência funcional, com aumento semelhante, independentemente do número de AVD nas quais o idoso necessitasse de auxílio para realização.

Quanto ao comprometimento da cognição, a prevalência de fragilidade aumentou de 1,9 vez (IC95\% 1,6-2,8) quando a reprovação ocorreu por erros mínimos. Houve, ainda, aumento da prevalência de fragilidade em 5,2 vezes (IC95\% 1,7-10,1), quando esta se deveu a erros significativos. Em relação ao comprometimento na realização de AVD, o aumento do número de atividades acompanhou o aumento da prevalência de fragilidade, passando de 3,3 vezes (IC95\% 1,6-8,4), quando o idoso requeria auxílio para realização, 2 a 4 atividades, para 4,8 vezes (IC95\% 2,9-24,0), quando a realização independente, de 5 a 8 atividades estava comprometida.

Observa-se que, entre os fatores avaliados pelo idoso, a autoavaliação de saúde, a frequência do suporte social, a percepção de perda de peso e o sentimento de tristeza/depressão interferiram na razão de prevalência exclusivamente. Note-se também que as variáveis 'escolaridade' e 'renda pessoal' não se mostraram significantes no modelo multivariado (tabela 4).

Tabela 4. Fatores associados à fragilidade segundo razão de prevalência bruta e ajustada de idosos residentes em Ilpi. Recife, PE, 2013

\begin{tabular}{|c|c|c|c|c|c|c|}
\hline $\begin{array}{l}\text { Variáveis relativas à síndrome da } \\
\text { fragilidade }\end{array}$ & $\begin{array}{r}\text { Amostra } \\
n\end{array}$ & $\begin{array}{l}\text { Frágil } \\
\mathrm{n}(\%)\end{array}$ & $\mathrm{RPb}(\mathrm{IC} 95 \%)$ & Valor $p$ & $\mathrm{RPa}(\mathrm{IC} 95 \%)$ & Valor $p$ \\
\hline Cognição & & & & $<0,001$ & & 0,002 \\
\hline Aprovado & 28 & $8(28,6)$ & 1,0 & & 1,0 & \\
\hline Reprovado com erros mínimos & 16 & $6(37,5)$ & $1,7(1,2-3,7)$ & & $1,9(1,6-2,8)$ & \\
\hline Reprovado com erros significativos & 170 & $136(80,0)$ & $5,1(1,6-9,4)$ & & $5,2(1,7-10,1)$ & \\
\hline Autoavaliação da saúdeł & & & & 0,001 & & $<0,001$ \\
\hline Excelente a boa & 88 & $42(47,7)$ & 1,0 & & 1,0 & \\
\hline Razoável & 79 & $63(79,7)$ & $2,7(1,8-10,2)$ & & $2,6(1,2-4,8)$ & \\
\hline Ruim & 46 & $44(95,7)$ & $14.4(2,8-24,3)$ & & $3,5(1,2-7,9)$ & \\
\hline Número de AVD^ comprometidas & & & & $<0,001$ & & 0,030 \\
\hline $0-1$ & 50 & $17(34,0)$ & 1,0 & & 1,0 & \\
\hline $2-4$ & 58 & $38(65,5)$ & $1,8(1,2-3,9)$ & & $3,3(1,6-8,4)$ & \\
\hline $5-8$ & 106 & $95(89,6)$ & $5,3(1,3-16,8)$ & & $5,0(2,9-24,0)$ & \\
\hline Disponibilidade de suporte social & & & & 0,005 & & 0,028 \\
\hline Sempre & 147 & $92(62,6)$ & 1,0 & & 1,0 & \\
\hline Algumas vezes & 45 & $40(88,9)$ & $3,5(1,2-7,9)$ & & $3,2(2,9-3,6)$ & \\
\hline Nunca & 22 & $18(81,8)$ & $2,6(1,0-4,9)$ & & $2,3(1,0-3,3)$ & \\
\hline
\end{tabular}


Tabela 4. (cont.)

\begin{tabular}{lrrrrr}
\hline $\begin{array}{l}\text { Autorreferência de perda de peso } \\
\text { recente }\end{array}$ & & & $\mathbf{0 , 0 0 1}$ & $\mathbf{0 , 0 0 1}$ \\
\hline Não & 129 & $77(59,7)$ & 1,0 & 1,0 \\
Sim & 80 & $70(87,5)$ & $2,1(1,8-2,6)$ & $1,9(1,7-2,2)$ \\
\hline Sentimento de tristeza/depressão & & & 0,005 & $<0,001$ \\
\hline Não & 94 & $48(51,1)$ & 1,0 & 1,0 \\
Sim & 120 & $102(85,0)$ & $2,3(1,8-3,8)$ & $3,1(1,7-5,0)$ \\
\hline
\end{tabular}

RPb: Razão de Prevalência Bruta; RPa: Razão de Prevalência Ajustada; łum (0,5\%) paciente omitiu esta informação; *AVD: Atividades de Vida Diária

\section{Discussão}

A prevalência de síndrome da fragilidade entre os idosos moradores em Ilpi no Recife, $\mathrm{PE}$, aponta para um quadro preocupante, asseverador de um problema de saúde pública a demandar atenção de pesquisadores e da sociedade em geral, especialmente por estarem esses idosos institucionalizados.

Ao se considerar o parâmetro de estatísticas para interpretação dos resultados neste estudo, verificou-se serem menores que os $63,7 \%$ constatados entre 240 idosos com 60 anos de idade ou mais, residindo em comunidade da cidade de Ribeirão Preto, SP, avaliados também pela escala de Edmonton?

A prevalência encontrada no presente estudo foi maior que o relatado na pesquisa FIBRA, realizada na cidade do Rio de Janeiro, RJ, assim como em sete cidades brasileiras, quando a prevalência de idosos frágeis igualou-se a 9,1 (IC95\% 7,3-11,3) ${ }^{8,9}$. No entanto, é preciso cautela nessa comparação já que os idosos residiam na comunidade, e o parâmetro de fragilidade adotado foi o de Fried et al. ${ }^{10}$ que difere da escala de Edmonton adotada na presente pesquisa, pela aferição da força de preensão, parâmetro mais frequentemente comprometido entre os idosos frágeis ${ }^{8,9}$.

Outro estudo que também apontou prevalência menor foi o realizado no Rio Grande do Norte, RN, incluindo 300 idosos com 65 anos de idade ou mais, residentes em comunidade, dos quais $23,8 \%$ eram frágeis $\mathbf{1}$.

A prevalência neste estudo foi menor que a relatada por Borges et al.5, que a referiram igual a $74,1 \%$ entre idosos institucionalizados em Fortaleza, CE, avaliados pela escala de Edmonton. Aos idosos frágeis, somaram-se $3,7 \%$ não frágeis e $22,2 \%$ vulneráveis ou pré-frágeis. Todavia, apesar da escala de fragilidade ter sido a mesma utilizada na presente pesquisa, houve diversas diferenças entre os idosos, entre as quais estiveram o predomínio de homens com 80 anos ou mais, maior tempo de institucionalização e menor nível de escolaridade.

Quanto aos estudos internacionais, a prevalência identificada nesta pesquisa foi menor. Castell et al."11, no norte de Madri, Espanha, relataram fragilidade em 10,5\% dos 1.327 idosos pesquisados, morando em comunidade e avaliados pela escala de Fried et al..$^{10}$. enquanto Solfrizzi et al.12 determinaram fragilidade em $7,6 \%$ dos 5.636 idosos italianos vivendo em comunidade e em Ilpi avaliados pela escala de Fried et al. ${ }^{12}$ modificada pela retirada da avaliação da força de preensão.

Colmenares et al. ${ }^{\mathbf{1 3}}$, estudando 311 idosos com 60 anos ou mais de idade, residindo em comunidades do Peru e triados a partir dos registros de consultas ambulatoriais de um centro médico, referiram serem frágeis $27,8 \%$. Em Cuba, em pesquisa envolvendo 2.813 idosos com 65 anos ou mais de idade, vivendo em comunidade, a prevalência de fragilidade igualou-se a 21,6 (IC95\% 17,9-23,8) ${ }^{\mathbf{1 4}}$. 
Com base nessas comparações, depreende-se que os idosos institucionalizados no Recife, PE, investigados por esta pesquisa, estavam expostos a menor risco de fragilidade que os idosos de Fortaleza, $\mathrm{CE}^{5}$, porém eram muito mais frágeis que os idosos residindo em outras comunidades nacionais, $\mathbf{1 , 5 , 7 - 9}$ e internacionais ${ }^{11-13}$. Todavia, a diversidade de metodologias empregadas nos estudos relativas ao ponto de corte de idade para classificar os participantes de pesquisa, à avaliação da síndrome de fragilidade e ao local de residência impede comparações mais acuradas.

Apesar dessa ressalva, percebe-se como relevante a alta prevalência de fragilidade entre os idosos desta pesquisa, por todas as consequências e riscos agregados à condição ${ }^{3-5,15}$. Eram idosos em condições sociais desfavoráveis, representadas pelo baixo nível de escolaridade, baixa renda e com autoavaliação de saúde predominante como razoável a ruim. Essa condição desfavorável os colocava em risco ainda maior de morbidades e de mortalidade, 3,10,16 considerando que a fragilidade é o reflexo de um continuum de vida, ao qual se somam os prejuízos próprios do envelhecimento e as perdas a ele inerentes.

Diante dessas questões, ressaltam-se os aspectos sociais e históricos da discussão dos resultados deste estudo. O Brasil é um país que ainda precisa equacionar questões pertinentes ao envelhecimento e aos idosos. Dessa forma, as Ilpi, como instituições que sofrem influência de suas relações internas e externas, repetem, pelo menos em parte, os costumes sociais e históricos do trato com idosos, o que contribui para sua fragilidade. As participações sociais de idosos são escassas mesmo para aqueles que vivem em comunidade, porque não são considerados como membros ativos da família e da sociedade. Para isso, contribui a restrição de sua capacidade laboral a auxiliar em atividades menores ou a suprir necessidades que a falta de tempo impõe aos membros mais jovens, como pagar contas ou buscar os netos na escola ${ }^{5,17,18}$.

Um ponto relevante nesta pesquisa foi verificar a importância do emprego da escala para avaliação da fragilidade. Nota-se que ao considerar os dados isolados dos componentes da escala de fragilidade, pode-se afirmar que os idosos pesquisados estavam com boa condição de saúde e expostos a baixo risco de morbidade e mortalidade. Apresentavam poucos internamentos, tinham suporte social frequente, não necessitavam de muitas medicações, não esqueciam de tomá-las, não perdiam peso, eram continentes e tinham bom desempenho funcional.

No entanto, ao compor o índice de fragilidade pela escala de Edmonton, o que se verificou foi uma alta prevalência de fragilidade, a qual deve guiar os cuidados a serem dedicados aos idosos de forma mais específica e individualizada. Para essa constatação, não se encontrou qualquer referência na literatura consultada, podendo ser esta uma recomendação útil para as Ilpi, no sentido de prestar uma atenção de melhor qualidade aos idosos.

Observa-se que neste estudo o nível de escolaridade não se comportou como fator de risco para a síndrome da fragilidade, contrariando o que se tem como consenso na literatura ${ }^{3,10}$.

Torna-se plausível que essa diferença possa ter resultado do fato do nível de escolaridade de idosos no Brasil ser baixo, posto que os esforços de alfabetização e escolarização foram foco de atenção do governo, principalmente a partir das últimas décadas do século passado ${ }^{19}$. Todavia, essa uniformidade de um padrão de escolarização menor é preocupante e pode, pelo menos em parte, justificar a frequência alta de distúrbios de cognição entre os idosos analisados.

A escolarização contribui para a formação da reserva cognitiva e permite ao indivíduo maior resiliência, ou seja, maior e melhor adequação às inúmeras situações de estresse que a vida em sociedade impõe ${ }^{1,20}$. A baixa reserva cognitiva confere aos idosos aumento da vulnerabilidade e os deixa mais expostos aos riscos de comprometimento da saúde; essa foi uma condição que homogeneizou os idosos analisados nesta pesquisa. 
Comprovou-se, ainda, que essas características cognitivas atuaram aumentando a prevalência da fragilidade isoladamente, que se constatou na análise bivariada, bem como de forma associada, como se detectou na análise multivariada. Esse conhecimento é útil para dedicar maior cuidado aos idosos com baixa renda, baixa escolaridade, longo tempo de moradia nas Ilpi, afastados do convívio de familiares e amigos, mesmo que ocasionalmente, vivenciando sentimentos de tristeza ou depressão.

Vale ressaltar, ainda, que fatores como um atendimento parcial à regulamentação vigente tanto nos aspectos físico-estruturais quanto organizacionais leva os idosos à exposição a um ambiente, por vezes, insalubre e a fatores de riscos para agravos à saúde que podem colaborar para a progressão e para intercorrências relacionadas com a fragilidade. Ressalta-se, assim, a importância de uma equipe multiprofissional para o cuidado integral às pessoas idosas institucionalizadas ${ }^{\mathbf{2 1}}$.

Muitas vezes, o fato de oferecer o básico (moradia, comida e higiene) é visto como o suficiente por parcela dos idosos institucionalizados ${ }^{22}$. Contudo, o acesso a um acompanhamento de saúde adequado é fundamental e condição precípua para identificar e melhor cuidar de idosos frágeis.

Pode-se identificar como limitação do estudo a perda de informações em algumas entrevistas que ficaram incompletas, sem o teste do relógio, devido à baixa escolaridade de parte da população investigada. Houve, ainda, limitação na análise estatística, por exemplo, da renda pessoal, cujo intervalo de confiança de $95 \%$ incluiu o valor 1,0 .

\section{Conclusões}

Considera-se, assim, que os achados apresentados podem contribuir para o direcionamento dos cuidados aos idosos residentes em instituições de longa permanência com especial atenção aos idosos com deficit cognitivo, tendo em vista suas maiores necessidades por serem mais frágeis.

É importante, também, que sejam discutidos em sociedade os cuidados a esses idosos frágeis, que estarão sob responsabilidade da família e/ou do Estado, estejam eles no ambiente doméstico ou em instituições de longa permanência. 


\section{Referências}

1. Amaral FLJS, Guerra, RO, Nascimento AFF, et al. Apoio social e síndrome da fragilidade em idosos residentes na comunidade. Ciênc. Saúde Colet. 2013; 18(5):1835-1846.

2. Lu Y, Tan CT, Nyunt MS, et al. Inflammatory and immune markers associated with physical frailty syndrome: findings from Singapore longitudinal aging studies. Oncotarget. 2016; 17(20):2878328795.

3. Chen X, Mao G, Leng SX. Frailty syndrome: an overview. Clinical Interventions in Aging. 2014; 9:433-441.

4. Fabrício SCC, Rodrigues RAP. Revisão da literatura sobre fragilidade e sua relação com o envelhecimento. Revista RENE. 2008; 9(2):113-119.

5. Borges CL, Silva MJ, Clares JWB, et al. Avaliação da fragilidade de idosos institucionalizados. Acta Paul. Enferm. 2013; 26(4):318-322.

6. Rolfson DB, Majundar SR, Tsuyuki RT, et al. Rockwood KL. Validity and reliability of Edmonton Frail Scale. Age Ageing. 2006; 35(5):523-526.

7. Fhon JR, Rosset I, Freitas CP, et al. Prevalence of falls among frail elderly adults. Rev. Saúde Pública. 2013; 47(2):266-273.

8. Moreira VG, Lourenço RA. Prevalence and factors associated with frailty in an older population from the city of Rio de Janeiro, Brazil: the FIBRA-RJ Study. Clinics. 2013; 68(7):979-985.

9. Neri AL, Yassuda MS, Araújo LF, et al. Metodologia e perfil sociodemográfico, cognitivo e de fragilidade de idosos comunitários de sete cidades brasileiras: Estudo FIBRA. Cad. Saúde Pública. 2013; 29(4):778792.

10. Fried LP, Tangen CM, Walston J, et al. Frailty in older adults: evidence for a phenotype. J Gerontol A Biol Sci Med Sci. 2001; 56(3):M146-56.
11. Castell MV, Sánchez M, Julián R, et al. Frailty prevalence and slow walking speed in persons age 65 and older: implications for primary care. BMC Fam Practi. 2013; 14(1):86-95.

12. Solfrizzi V, Scafato E, Frisard V, et al. Frailty syndrome and all-cause mortality in demented patients: the Italian Longitudinal Study on Aging. Age (Dordr). 2012; 34(2):507-517.

13. Colmenares FMR, Samper-Ternent R, Al Snih S, et al. Prevalence and factors associated with frailty among peruvian older adults. Arch Gerontol Geriatr. 2014; 58(1):1-11.

14. Llibre JDEJ, López AM, Valhuerdi A, et al. Frailty, Dependency and Mortality Predictors in a Cohort of Cuban Older Adults, 2003-2011. MEDIC Rev. 2014; 16(1):24-30.

15. Calado LB, Ferrioli E, Moriguti JC, et al. Frailty syndrome in an independent urban population in Brazil (FIBRA study): a cross-sectional populational study. São Paulo Med. J. 2016; 134(5):385-392.

16. Bergman H, Ferruci L, Guralnik J, et al. Frailty: an emerging research and clinical paradigm-issues and controversies. J Gerontol A Biol Sci Med Sci. 2009 jul; 62(7):731-737.

17. Farias RG, Santos SMA. Influência dos determinantes do envelhecimento ativo entre idosos mais idosos. Texto \& Contexto Enferm. 2012; 21(2)167-176.

18. Gobbens RJ, Van Assen MA, Luijkx KG, et al. Determinants of frailty. J Am Med Dir Assoc. 2010 jun; 11(5):356-364.

19. Lima CRV. Políticas públicas para idosos: a realidade das instituições de longa permanência no Distrito Federal. Brasília, DF: Câmara dos Deputados; 2011.

20. Mello JA, Van Durme T, Macq J, et al. Interventions to delay institutionalization of frail older persons: 
design of a longitudinal study in the home care setting. BMC Public Health. 2012; 12(1):615-621.

21. Alves MB, Menezes MR, Felzemburg RDM, et al. Instituições de longa permanência para idosos: aspectos físico-estruturais e organizacionais. Esc. Anna Nery. 2017;21(4):1-8.
22. Oliveira JM, Rozendo CA. Instituição de longa permanência para idosos: um lugar de cuidado para quem não tem opção. Rev Bras Enferm. 2014; 67(5):773-779.

Recebido em 16/09/2017

Aprovado em 28/04/2018

Conflito de interesses: inexistente

Suporte financeiro: não houve 